\title{
Mixed state geometric phases, entangled systems, and local unitary transformations
}

\author{
Marie Ericsson, ${ }^{1}$ Arun K. Pati, ${ }^{2}$ Erik Sjöqvist, ${ }^{1}$ Johan Brännlund, ${ }^{3}$ and Daniel. K. L. Oi ${ }^{4}$ \\ ${ }^{1}$ Department of Quantum Chemistry, Uppsala University, Box 518, Se-751 20 Sweden \\ ${ }^{2}$ Institute of Physics, Bhubaneswar-751005, Orissa, India \\ ${ }^{3}$ SCFAB, Department of Physics, Stockholm University, Se-106 91 Stockholm, Sweden \\ ${ }^{4}$ Centre for Quantum Computation, Clarendon Laboratory, \\ University of Oxford, Parks Road, Oxford OX1 3PU, UK
}

\begin{abstract}
The geometric phase for a pure quantal state undergoing an arbitrary evolution is a "memory" of the geometry of the path in the projective Hilbert space of the system. We find that Uhlmann's geometric phase for a mixed quantal state undergoing unitary evolution not only depends on the geometry of the path of the system alone but also on a constrained bi-local unitary evolution of the purified entangled state. We analyze this in general, illustrate it for the qubit case, and propose an experiment to test this effect. We also show that the mixed state geometric phase proposed recently in the context of interferometry requires uni-local transformations and is therefore essentially a property of the system alone.
\end{abstract}

PACS numbers: 03.65.Vf, 42.50.Dv

Pancharatnam 1] was first to introduce the concept of geometric phase in his study of interference of light in distinct states of polarization. Its quantal counterpart was discovered by Berry [2], who proved the existence of geometric phases in cyclic adiabatic evolutions. This was generalized to the case of nonadiabatic [3] and noncyclic 4] evolutions. The geometric phase was also derived on the basis of purely kinematic considerations [5]. In a general context, the geometric phase was defined for nonunitary and non-Schrödinger [ [] $]$ evolutions. Since the geometric phase for a pure state is a nonintegrable quantity and depends only on the geometry of the path traced in the projective Hilbert space, it acts as a memory of a quantum system.

Another important development in this field was initiated by Uhlmann [7] (see also [8]), who introduced a notion of geometric phase for mixed quantal states. More recently, using ideas of interferometry, another definition of mixed state phase was introduced in [9] (see also [10]) and experimentally verified in 11]. A renewed interest in geometric phases for mixed states is due to its potential relevance to geometric quantum computation [12].

Mixed states naturally arise when we ignore the ancilla subsystem of a composite object (system+ancilla) that is described by a pure entangled state. In this Letter we wish to consider the mixed state geometric phases in [7, 9] in terms of such purifications, and to investigate whether they should be regarded as properties of the system alone or not. More precisely, we would like to address the following question: do mixed state geometric phases depend only on the evolution of the system of interest, or do they also depend on the evolution of the ancilla part with which the system is entangled? By examining, in detail, the case of mixed states undergoing local unitary evolutions, we find that the Uhlmann phase [7] indeed contains a memory of the ancilla part, while the mixed state phase proposed in [9] does not. In particular, we propose an experiment to test the Uhlmann phase using a Franson set up [13] with polarization entangled photons [14, 15] that would verify this new memory effect. More importantly, we show that the phase holonomies given in [7] and in [9] are generically different.

Consider first the unitary path $\eta: t \in[0, \tau] \mapsto\left|\psi_{t}\right\rangle\left\langle\psi_{t}\right|$ of normalized pure state projectors with $\left\langle\psi_{0} \mid \psi_{\tau}\right\rangle \neq 0$. The geometric phase associated with $\eta$ is defined as

$\beta=\arg \lim _{N \rightarrow \infty}\left(\left\langle\psi_{0} \mid \psi_{\tau}\right\rangle\left\langle\psi_{\tau} \mid \psi_{[(N-1) \tau / N]}\right\rangle \times \ldots \times\left\langle\psi_{[\tau / N]} \mid \psi_{0}\right\rangle\right)$

$\beta$ is a property only of the path $\eta$ as it is independent of the lift $\eta \longrightarrow \tilde{\eta}: t \in[0, \tau] \mapsto\left|\psi_{t}\right\rangle$. A parallel lift is defined by requiring that each $\left\langle\psi_{[(j+1) \tau / N]} \mid \psi_{[j \tau / N]}\right\rangle$ be real and positive (i.e. $\langle\psi \mid \dot{\psi}\rangle=0$ when $N \rightarrow \infty$ ), so that $\beta$ takes the form

$$
\beta=\arg \left\langle\psi_{0} \mid \psi_{\tau}\right\rangle .
$$

One may measure $\beta$ in interferometry as a relative phase shift in the interference pattern characterized by $\nu e^{i \beta}=$ $\left\langle\psi_{0} \mid \psi_{\tau}\right\rangle$, where $\nu=\left|\left\langle\psi_{0} \mid \psi_{\tau}\right\rangle\right|$ is the visibility [16].

To generalize the above to mixed states, consider the path $\zeta: t \in[0, \tau] \longrightarrow \rho_{t}$ of density operators $\rho_{t}$. A standard purification (lift) of $\zeta$ is a path $\tilde{\zeta}: t \in$ $[0, \tau] \longrightarrow w_{t}$ in the Hilbert space of Hilbert-Schmidt operators with scalar product $\left\langle w_{t}, w_{t^{\prime}}\right\rangle=\operatorname{Tr}\left(w_{t}^{\dagger} w_{t^{\prime}}\right)$ such that $w_{t} w_{t}^{\dagger}=\rho_{t}$. Note that $w_{t}=\rho_{t}^{1 / 2} x_{t}$ is a purification of $\rho_{t}$ for any unitary $x_{t}$. For a purification where each $\left|\left\langle w_{t}, w_{t^{\prime}}\right\rangle\right|$ is constrained to its maximum $d\left[\rho_{t}, \rho_{t^{\prime}}\right]_{\text {Bures }}=\operatorname{Tr}\left[\sqrt{\sqrt{\rho_{t}} \rho_{t^{\prime}} \sqrt{\rho_{t}}}\right]$ 17], Uhlmann [7] defines the geometric phase associated with $\zeta$ as

$$
\begin{aligned}
\phi_{g}= & \arg \lim _{N \rightarrow \infty}\left(\left\langle w_{0}, w_{\tau}\right\rangle\left\langle w_{\tau}, w_{[(N-1) \tau / N]}\right\rangle\right. \\
& \left.\times \ldots \times\left\langle w_{[\tau / N]}, w_{0}\right\rangle\right) .
\end{aligned}
$$

The Uhlmann phase $\phi_{g}$ is independent of the purification $\zeta \longrightarrow \tilde{\zeta}$ as long as it obeys the maximality constraint, 
thus $\phi_{g}$ is a property of the path $\zeta$. For pure states $\rho_{t}=\left|\psi_{t}\right\rangle\left\langle\psi_{t}\right|$ the constrained purification is characterized by $\left\langle w_{t}, w_{t^{\prime}}\right\rangle=\left\langle\psi_{t} \mid \psi_{t^{\prime}}\right\rangle$ up to an arbitrary phase factor so that $\phi_{g}$ reduces to the pure state geometric phase $\beta$. A parallel purification is introduced by requiring that each $w_{[(j+1) \tau / N]}^{\dagger} w_{[j \tau / N]}$ be hermitian and positive for all $j=0, \ldots, N$. Infinitesimally, this entails that

$$
w_{t}^{\dagger} \dot{w}_{t}=\dot{w}_{t}^{\dagger} w_{t}
$$

For such a parallel purification, the geometric phase becomes

$$
\phi_{g}=\arg \left\langle w_{0}, w_{\tau}\right\rangle
$$

which reduces to $\beta$ for pure states. We show below that $\phi_{g}$ could be verified in interferometry as a relative phase shift in the interference pattern characterized by the visibility $\left|\left\langle w_{0}, w_{\tau}\right\rangle\right|$.

To elucidate the above purification approach, consider the unitary case $\rho_{0} \longrightarrow \rho_{t}=u_{t} \rho_{0} u_{t}^{\dagger}$. We introduce a set of eigenvectors $\{|k\rangle\}, k=1, \ldots, N$ with $N$ the (finite) dimension of Hilbert space, with eigenvalues $\left\{\lambda_{k}\right\}$ of $\rho_{0}$ so that

$$
\begin{aligned}
w_{0} & =\rho_{0}^{1 / 2}=\sum_{k} \sqrt{\lambda_{k}}|k\rangle\langle k| \\
\longrightarrow w_{t} & =u_{t} \rho_{0}^{1 / 2} v_{t}=\sum_{k} \sqrt{\lambda_{k}} u_{t}|k\rangle\langle k| v_{t}
\end{aligned}
$$

with the unitarity $v_{t}=u_{t}^{\dagger} x_{t}$. With $u_{t}$ and $v_{t}$ related via the parallel transport condition Eq. (4), we obtain the geometric phase from Eq. (5) as

$$
\phi_{g}=\arg \sum_{k, l} \sqrt{\lambda_{k} \lambda_{l}}\left\langle l\left|u_{\tau}\right| k\right\rangle\left\langle k\left|v_{\tau}\right| l\right\rangle .
$$

The standard purification used by Uhlmann is equivalent to considering a pure state of the system + ancilla, $w_{0} \longleftrightarrow$ $\left|\Psi_{0}\right\rangle \in \mathcal{H}_{s} \otimes \mathcal{H}_{a}$ evolving under a bi-local operator $u_{t} \otimes y_{t}$, in Schmidt form,

$$
w_{t} \longleftrightarrow\left|\Psi_{t}\right\rangle=\sum_{k} \sqrt{\lambda_{k}}\left(u_{t}|k\rangle\right) \otimes\left(y_{t}|k\rangle\right),
$$

where the ancilla unitary $y_{t}=v_{t}^{T}$ (transpose with respect to the instantaneous eigenbasis of $\rho_{t}$ ) obeys the same parallel condition as before. In this view the geometric phase is given by

$$
\phi_{g}=\arg \left\langle\Psi_{0} \mid \Psi_{\tau}\right\rangle \text {. }
$$

Let us now consider the case where the composite system undergoes uni-local unitary transformations so that only the 'system' part is affected, i.e. unitarities of the form $u_{t} \otimes \mathbf{1}$. The purified state now evolves to

$$
\left|\Psi_{t}\right\rangle=\sum_{k} \sqrt{\lambda_{k}}\left(u_{\tau}|k\rangle\right) \otimes|k\rangle
$$

and the phase difference between the initial and final state reads

$$
\arg \left\langle\Psi_{0} \mid \Psi_{\tau}\right\rangle=\arg \sum_{k} \lambda_{k}\left\langle k\left|u_{\tau}\right| k\right\rangle=\arg \operatorname{Tr}\left[\rho_{0} u_{\tau}\right] .
$$

If we require $u_{t}$ to transport each pure state component $|k\rangle$ of the density matrix in a parallel manner, then

$$
\Phi_{g}=\arg \sum_{k} \lambda_{k} \nu_{k} e^{i \beta_{k}}
$$

where $\left\langle k\left|u_{\tau}\right| k\right\rangle=\nu_{k} e^{i \beta_{k}}$ and $\beta_{k}$ is the pure state (noncyclic) geometric phase for $|k\rangle . \Phi_{g}$ is the mixed state geometric phase proposed in [9].

It is natural to ask when the two mixed state geometric phases match. To see this, let us write $u_{t}=\exp (-i t H)$ and $v_{t}=\exp (i t \tilde{H}), H$ and $\tilde{H}$ being the Hamiltonian of system and ancilla, respectively (we set $\hbar=1$ ). The Hamiltonians $H$ and $\tilde{H}$ are both assumed to be timeindependent. To determine $\tilde{H}$ from the parallel transport condition Eq. (4), we write $\rho_{0}$ in its diagonal basis yielding 18

$$
\tilde{H}=\sum_{k, l} \frac{2 \sqrt{\lambda_{k} \lambda_{l}}}{\lambda_{k}+\lambda_{l}}|k\rangle\langle l|\langle k|H| l\rangle .
$$

Now, $v_{t}=\mathbf{1}$ iff $\tilde{H}=0$, which implies that $H=0$ when all $\lambda_{k}$ are nonvanishing. That is, when all $\lambda_{k} \neq 0$ the two geometric phases can match only in the trivial case where neither the system nor ancilla evolve. Thus, in generic cases the two phases are distinct and one cannot obtain one from the other. However, if $\rho$ is not of full rank, $\tilde{H}=0$ does not imply $H=0$ in order to match the two geometric phases. Only in the extreme case of $\rho$ being pure, the two geometric phases are identical and equal to the standard geometric phase of the system.

It can be seen that Uhlmann's geometric phase is in general a property of a composite system in a pure entangled state that undergoes a certain bi-local unitary transformation. Hence, this geometric phase depends on the history of the system as well as on the history of its entangled counterpart. On the other hand the geometric phase proposed in [9] requires that the entangled composite system undergoes a uni-local unitary transformation, i.e. the evolution of the ancilla is independent of the evolution of the system. Thus, this geometric phase is essentially a property of the system alone; the role of the ancilla is just to make the reduced state of the system mixed.

It should be noted that the above memory effects are not equivalent to that of the standard geometric phase acquired by the purified state, as computed in Ref. [19]. In fact, the parallelity condition $\left\langle\Psi_{t} \mid \dot{\Psi}_{t}\right\rangle=0$ on the purified state is a much weaker constraint on the bilocal transformation than Eq. (4). Indeed, by writing 
$\left|\Psi_{t}\right\rangle=u_{t} \otimes y_{t}\left|\Psi_{0}\right\rangle$ the parallel transport constitutes a single condition

$$
\left\langle\Psi_{0}\left|u_{t}^{\dagger} \dot{u}_{t} \otimes \mathbf{1}\right| \Psi_{0}\right\rangle+\left\langle\Psi_{0}\left|\mathbf{1} \otimes y_{t}^{\dagger} \dot{y}_{t}\right| \Psi_{0}\right\rangle=0,
$$

and there are infinitely many $y_{t}$ that fulfill Eq. (14) but not Eq. (4). For uni-local transformations Eq. (14) reduces to

$$
\left\langle\Psi_{0}\left|u_{t}^{\dagger} \dot{u}_{t} \otimes \mathbf{1}\right| \Psi_{0}\right\rangle=0,
$$

which is also a weaker condition than that for $\Phi_{g}$. In fact, $\Phi_{g}$ requires that each $\left\langle k\left|u_{t}^{\dagger} \dot{u}_{t}\right| k\right\rangle$ associated with nonvanishing $\lambda_{k}$ does vanish, while in Eq. (15) only their sum vanishes. Only for $|\Psi\rangle$ being a product state, corresponding to a pure state of the system, the new memory effects match with the standard geometric phase.

Let us now compute Uhlmann's geometric phase in the noncyclic case for a qubit (two-level system) undergoing unitary precession. We assume that the qubit's Bloch vector initially points in the $z$ direction and has length $r$ so that $\rho_{0}$ has eigenvalues $\frac{1}{2}(1 \pm r)$. Furthermore, assume that the Hamiltonian of the system is $H=\frac{1}{2} \boldsymbol{n} \cdot \boldsymbol{\sigma}=\frac{1}{2}\left(n_{x} \sigma_{x}+n_{z} \sigma_{z}\right),|\boldsymbol{n}|^{2}=n_{x}^{2}+n_{z}^{2}=1$. This determines the Hamiltonian $\tilde{H}$ of the ancilla via Eq. (13) as $\tilde{H}=\frac{1}{2}\left(\sqrt{1-r^{2}} n_{x} \sigma_{x}+n_{z} \sigma_{z}\right)$. By introducing the unit vector $\tilde{\boldsymbol{n}}=\left(\tilde{n}_{x}, 0, \tilde{n}_{z}\right)$ with the components $\tilde{n}_{x}=\sqrt{1-r^{2}} n_{x} / \sqrt{1-r^{2} n_{x}^{2}}, \tilde{n}_{z}=n_{z} / \sqrt{1-r^{2} n_{x}^{2}}$, and the parameter $\tilde{\tau}=\tau \sqrt{1-r^{2} n_{x}^{2}}$ we obtain the noncyclic Uhlmann phase as

$$
\begin{aligned}
\phi_{g}= & -\arctan \left(\left(r n_{z} \tan \frac{\tau}{2}-r \tilde{n}_{z} \tan \frac{\tilde{\tau}}{2}\right) /\left(1+\left(n_{z} \tilde{n}_{z}\right.\right.\right. \\
& \left.\left.\left.+\sqrt{1-r^{2}} n_{x} \tilde{n}_{x}\right) \tan \frac{\tau}{2} \tan \frac{\tilde{\tau}}{2}\right)\right) .
\end{aligned}
$$

Let us consider some important special cases. Firstly, the cyclic Uhlmann phase is obtained by inserting $\tau=2 \pi$ and using $-\tan x=\tan (\pi-x)$ yielding

$$
\phi_{g}=\arctan \left(\frac{r n_{z}}{\sqrt{1-r^{2} n_{x}^{2}}} \tan \left(\pi \sqrt{1-r^{2} n_{x}^{2}}\right)\right) .
$$

Secondly, in the noncyclic pure state case $(r=1)$, we have $\tilde{\boldsymbol{n}}=(0,0,1)$ and $\sqrt{1-n_{x}^{2}}=\left|n_{z}\right|$, which yields

$$
\phi_{g}=-\arctan \left(n_{z} \tan (\tau / 2)\right)+\frac{\tau}{2} n_{z} \bmod 2 \pi .
$$

This equals minus one-half of the geodesically closed solid angle of the open path on the Bloch sphere and is consistent with known expression for the geometric phase in the case of a pure qubit undergoing noncyclic precession (see, e.g., Ref. 20]). Finally, in the case of the maximally mixed state $(r=0), \tilde{\boldsymbol{n}}=\boldsymbol{n}$ and $\rho_{0}^{1 / 2}=\mathbf{1} / \sqrt{2}$, which yields $w_{0} w_{\tau}^{\dagger}=\rho_{0}$ so that the geometric phase vanishes, i.e. $\phi_{g}=\arg \operatorname{Tr} \rho_{0}=0$.

Let us now compare the above results with the mixed state geometric phase in [9]. In the diagonal basis

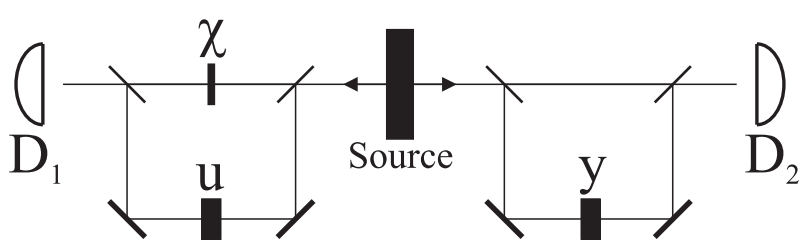

FIG. 1: Two-photon interferometry set up to test the Uhlmann phase.

$\{|0\rangle,|1\rangle\}$ of $\rho_{0}$ we have $\nu_{0}=\nu_{1}$ and $\beta_{0}=-\beta_{1}=$ $-\frac{1}{2} \Omega$, where $\Omega$ is the geodesically closed solid angle on the Bloch sphere. For $r \neq 0$, we obtain $\Phi_{g}=$ $-\arctan (r \tan (\Omega / 2))$. These expressions become identical to those of the Uhlmann approach only for pure states and in the trivial case $\boldsymbol{n}=(0,0,1)$, where neither system nor ancilla evolve. In the maximally mixed case $\Phi_{g}$ is even indeterminate as the parallel transport conditions $\left\langle 0\left|u_{t}^{\dagger} \dot{u}_{t}\right| 0\right\rangle=\left\langle 1\left|u_{t}^{\dagger} \dot{u}_{t}\right| 1\right\rangle=0$ do not specify a unique $u_{t}$ for a degenerate density operator, making $\Phi_{g}=\arg \operatorname{Tr}\left[\rho_{0} u_{\tau}\right]=\arg \operatorname{Tr}\left[\frac{1}{2} u_{\tau}\right]$ undefined.

As is clear from Eq. (8), Uhlmann's geometric phase retains a memory of the evolution of both system and ancilla due to the parallelity condition Eq. (4). Using the above purification scheme $w_{t} \longrightarrow\left|\Psi_{t}\right\rangle$, the memory effect associated with $\phi_{g}$ could be tested experimentally in polarization entangled two-photon interferometry, as now shall be demonstrated. A detailed description of the relevant set up shown in Fig. (1) may be found in Ref. 14]. A photon pair (system and ancilla photon) is produced in a polarization entangled pure state that takes the Schmidt form in the horizontal-vertical $(H-V)$ basis:

$$
\left|\Psi_{0}\right\rangle=\sqrt{\frac{1+r}{2}}|H\rangle \otimes|H\rangle+\sqrt{\frac{1-r}{2}}|V\rangle \otimes|V\rangle .
$$

This source is described in Ref. [15], and is used as input in a Franson interferometer [13]. Note that $\rho_{0}=$ $\operatorname{Tr}_{a}\left|\Psi_{0}\right\rangle\left\langle\Psi_{0}\right|=\frac{1}{2}\left(1+r \sigma_{z}\right)$ in the $H-V$ basis, and that $\left|\Psi_{0}\right\rangle$ is isomorphic to $w_{0}=\sqrt{\frac{1+r}{2}}|H\rangle\left\langle H\left|+\sqrt{\frac{1-r}{2}}\right| V\right\rangle\langle V|$.

The two unitary operators $u_{\tau}$ and $y_{\tau}$ are applied to the two longer arms. Thus, $u_{\tau}$ is applied to the system photon, say, and $y_{\tau}$ is applied to the ancilla photon. In one of the shorter arms a $U(1)$ shift $\chi$ is applied. To observe interference of $\left|\Psi_{0}\right\rangle$ and $\left|\Psi_{\tau}\right\rangle=u_{\tau} \otimes v_{\tau}\left|\Psi_{0}\right\rangle$ we require that the source produces photon pairs randomly [13], as is the case with the present type of source. If the photons arrive in the detector pair simultaneously, they either both took the shorter path $\left(\Psi_{0}\right)$ or the longer path $\left(\Psi_{\tau}\right)$. The state detected in coincidence is the desired superposition $|\Psi\rangle \sim e^{i \chi}\left|\Psi_{0}\right\rangle+\left|\Psi_{\tau}\right\rangle$. The measured coincidence intensity is proportional to $\langle\Psi \mid \Psi\rangle \propto 1+\nu \cos \left(\chi-\phi_{g}\right)$, where the visibility is $\nu=\left|\left\langle\Psi_{0} \mid \Psi_{\tau}\right\rangle\right|$. Thus, by varying $\chi$ the Uhlmann phase $\phi_{g}$ could be tested using this two-photon set up. 
An explicit realization of the operators $u_{\tau}$ and $y_{\tau}$ could be constructed in terms of an appropriate pair of $\lambda$-plates as follows. The $S U(2)$ part of the effect in the $H-V$ basis of a $\lambda$-plate making an angle $\theta$ with the vertical $(V)$ axis is given by $u(\alpha, \theta)=\exp \left(-i \frac{\alpha}{2} \boldsymbol{n}_{\theta} \cdot \boldsymbol{\sigma}\right)$ with $\boldsymbol{n}_{\theta}=(\sin [2 \theta], 0, \cos [2 \theta])$. The precession angle $\alpha$ is proportional to the thickness of the $\lambda$-plate (e.g., $\alpha=\frac{\pi}{2}$ for a $\frac{\lambda}{4}$-plate). Now, the Uhlmann phase is obtained by taking $u_{\tau}=u(\alpha, \theta)$ and $y_{\tau}=u^{\dagger}(\tilde{\alpha}, \tilde{\theta})$, where the thickness and orientation of the two $\lambda$-plates are related as $\tilde{\alpha} / \alpha=\sqrt{1-r^{2} \sin ^{2}(2 \theta)}$ and $\tan (2 \tilde{\theta})=\sqrt{1-r^{2}} \tan (2 \theta)$.

In the cyclic case, $\alpha=2 \pi$ and the visibility of the interference pattern is reduced by the geometric factor

$$
\begin{aligned}
\nu & =\left(\cos ^{2}\left(\pi \sqrt{1-r^{2} \sin ^{2}(2 \theta)}\right)\right. \\
& \left.+\frac{r^{2} \cos ^{2}(2 \theta)}{1-r^{2} \sin ^{2}(2 \theta)} \sin ^{2}\left(\pi \sqrt{1-r^{2} \sin ^{2}(2 \theta)}\right)\right)^{1 / 2}
\end{aligned}
$$

Thus, the visibility is reduced by the entanglement of the purified state. For maximally mixed states, corresponding to maximally entangled $\Psi_{0}$ [21], $\tilde{\alpha}=\alpha$ and $\tilde{\theta}=\theta$ so that $y_{\tau}=u_{\tau}^{\dagger}(\alpha, \theta)$. Thus one should choose the same thickness of the two $\lambda$-plates and their half axes being perpendicular. The scalar product $\left\langle\Psi_{0}\left|u_{\tau} \otimes y_{\tau}\right| \Psi_{0}\right\rangle=$ $\left\langle\Psi_{0}\left|u_{\tau} \otimes u_{\tau}^{\dagger}\right| \Psi_{0}\right\rangle$ becomes real-valued and hence $\phi_{g}=0$. The absence of phase shift could, e.g., be tested by varying the common angle $\theta$. For pure states, $\tilde{\alpha}=\alpha \cos 2 \theta$ and $\tilde{\theta}=0 \bmod \frac{\pi}{2}$. This yields the pure state geometric phase $\phi_{g}=-\frac{1}{2} \Omega$, which also could be tested in singlephoton interferometry [16].

The mixed state geometric phase in $[9]$ could be tested by canceling the accumulation of local phase changes for each pure state component in each beam of a singlephoton interferometer. Thus, if one of the beams is exposed to the unitarity $u_{t}$, the other beam should be exposed to the unitarity $\tilde{u}_{t}$ fulfilling $\left\langle 0\left|\tilde{u}_{t}^{\dagger} \dot{\tilde{u}}_{t}\right| 0\right\rangle=$ $-\left\langle 0\left|u_{t}^{\dagger} \dot{u}_{t}\right| 0\right\rangle$ and $\left\langle 1\left|\tilde{u}_{t}^{\dagger} \dot{\tilde{u}}_{t}\right| 1\right\rangle=-\left\langle 1\left|u_{t}^{\dagger} \dot{u}_{t}\right| 1\right\rangle[16]$.

To conclude, we have shown that the mixed state geometric phases proposed in [7] and [9] can be interpreted as two types of generically distinct phase holonomy effects for entangled systems undergoing certain local unitary transformations. We have shown that these phase effects are different from the standard geometric phase of the purified state. In the unitary case, the Uhlmann phase depends on the path of the system as well as on the ancilla undergoing a constrained bi-local unitary operation. This is a new type of memory effect that is present only for mixed state phase holonomy. We have proposed an experiment using polarization entangled photons to test this effect. The geometric phase in [9] depends on a certain uni-local transformation in which the ancilla part does not evolve. Thus, this geometric phase is essentially a property of the system part alone and is testable in one-particle interferometry. We hope that the mixed state phases would have applications in many areas of physics and future experiments would test these memory effects.

We would like to thank Artur Ekert for useful suggestions. The work by E.S. was financed by the Swedish Research Council. D.K.L.O acknowledges the support of CESG (UK) and QAIP grant IST-1999-11234.

[1] S. Pancharatnam, Proc. Indian Acad. Sci. A 44, 247 (1956).

[2] M. V. Berry, Proc. R. Soc. London Ser. A 392, 457 (1984).

[3] Y. Aharonov and J.S. Anandan, Phys. Rev. Lett. 58, 1593 (1987)

[4] J. Samuel and R. Bhandari, Phys. Rev. Lett. 60, 2339 (1988).

[5] N. Mukunda and R. Simon, Ann. Phys. (N.Y.) 228, 205 (1993).

[6] A.K. Pati, Phys. Rev. A 52, 2576 (1995); A.K. Pati, J. Phys. A 28, 2087 (1995).

[7] A. Uhlmann, Rep. Math. Phys. 24, 229 (1986).

[8] A. Uhlmann, Ann. Phys. (Leipzig) 46, 63 (1989). A. Uhlmann, Lett. Math. Phys. 21, 229 (1991); M. Hübner, Phys. Lett. A 179, 226 (1993); A. Uhlmann, J. Geom. Phys. 18, 76 (1996).

[9] E. Sjöqvist, A.K. Pati, A. Ekert, J.S. Anandan, M. Ericsson, D.K.L. Oi, and V. Vedral, Phys. Rev. Lett. 85, 2845 (2000).

[10] R. Bhandari, Phys. Rev. Lett 89, 268901 (2002); J.S. Anandan, E. Sjöqvist, A.K. Pati, A. Ekert, M. Ericsson, D.K.L. Oi, and V. Vedral, Phys. Rev. Lett 89, 268902 (2002); P.B. Slater, Lett. Math. Phys. 60, 123 (2002); e-print math-ph/0112054

[11] J.F. Du, P. Zou, M. Shi, L.C. Kwek, J.W. Pan, C.H. Oh, A. Ekert, D.K.L. Oi, and M. Ericsson, e-print quant-ph/0305054

[12] P. Zanardi and M. Rasetti, Phys. Lett. A 264, 94 (1999); J. Pachos, P. Zanardi, and M. Rasetti, Phys. Rev. A 61, R010305 (2000); J.A. Jones, V. Vedral, A. Ekert, and G. Castagnoli, Nature (London) 403, 869 (2000); A. Ekert, M. Ericsson, P. Hayden, H. Inamori, J.A. Jones, D.K.L. Oi, and V. Vedral, J. Mod. Opt. 47, 2501 (2000); W. Xiang-Bin and M. Keiji, Phys. Rev. Lett. 87, 097901 (2001); L.M. Duan, J.I. Cirac, and P. Zoller, Science 292, 1695 (2001); D. Ellinas and J. Pachos, Phys. Rev. A 64, 022310 (2001).

[13] J. D. Franson, Phys. Rev. Lett. 62, 2205 (1989).

[14] B. Hessmo and E. Sjöqvist, Phys. Rev. A 62, 062301 (2000).

[15] A.G. White, D.F.V. James, P.H. Eberhard, and P.G. Kwiat, Phys. Rev. Lett. 83, 3103 (1999).

[16] E. Sjöqvist, Phys. Lett. A 286, 4 (2001).

[17] A. Uhlmann, Rep. Math. Phys. 9, 273 (1976).

[18] A. Uhlmann, in Symmetry in Physics V, Algebraic Systems, Their Representations, Realizations, and Physical Applications, Ed. B. Gruber (Plenum Press, New York, 1993);

[19] E. Sjöqvist, Phys. Rev. A 62, 022109 (2000). 
[20] D.N. Klyshko, Phys. Lett. A 140, 19 (1989).

[21] A two-photon experiment demonstrating geometric phase effects for maximally entangled states was carried out in: D.V. Strekalov and Y.H. Shih, Phys. Rev. A 56, 3129 (1997). Although this experiment has some features in common to our proposal it should be noted that the unitary operations on the photons in Strekalov and Shih's experiment are not related in order to fulfill Uhlmann's parallel transport condition and therefore are not measuring Uhlmann's geometric phase. 